\title{
Downsizing Treatment with Tyrosine Kinase Inhibitors in Patients with Advanced Gastrointestinal Stromal Tumors Improved Resectability
}

\author{
Katarina Sjölund • Anna Andersson • \\ Erik Nilsson • Ola Nilsson • Håkan Ahlman • \\ Bengt Nilsson
}

Published online: 29 May 2010

(c) The Author(s) 2010. This article is published with open access at Springerlink.com

\begin{abstract}
Background Gastrointestinal stromal tumors (GISTs) express the receptor tyrosine kinase KIT. Most GISTs have mutations in the KIT or PDGFRA gene, causing activation of tyrosine kinase. Imatinib, a tyrosine kinase inhibitor (TKI), is the first-line palliative treatment for advanced GISTs. Sunitinib was introduced for patients with mutations not responsive to imatinib. The aim was to compare the survival of patients with high-risk resected GISTs treated with TKI prior to surgery with historical controls and to determine if organ-preserving surgery was facilitated.

Methods Ten high-risk GIST-patients had downsizing/ adjuvant TKI treatment: nine with imatinib and one with sunitinib. The patients were matched with historical controls $(n=89)$ treated with surgery alone, from our population-based series $(n=259)$. Mutational analysis of KIT and PDGFRA was performed in all cases. The progressionfree survival was calculated.

Results The primary tumors decreased in mean diameter from $20.4 \mathrm{~cm}$ to $10.5 \mathrm{~cm}$ on downsizing imatinib. Four patients with $\mathrm{R} 0$ resection and a period of adjuvant imatinib had no recurrences versus $67 \%$ in the historical control group. Four patients with residual liver metastases have
\end{abstract}

\footnotetext{
K. Sjölund · A. Andersson · E. Nilsson · H. Ahlman ·

B. Nilsson $(\square)$

Department of Surgery, Sahlgrenska Academy at the University

of Gothenburg, Sahlgrenska University Hospital, 41345

Gothenburg, Sweden

e-mail: bengt.e.nilsson@vgregion.se

\section{O. Nilsson}

Department of Pathology, Sahlgrenska Academy at the University of Gothenburg, Sahlgrenska University

Hospital, 41345 Gothenburg, Sweden
}

stable disease on continuous imatinib treatment after surgery. One patient has undergone reoperation with liver resection. The downsizing treatment led to organ-preserving surgery in nine patients and improved preoperative nutritional status in one patient.

Conclusions Downsizing TKI is recommended for patients with bulky tumors with invasion of adjacent organs. Sunitinib can be used for patients in case of imatinib resistance (e.g., wild-type GISTs), underlining the importance of mutational analysis for optimal surgical planning.

\section{Introduction}

Surgery is the primary treatment for nonmetastatic gastrointestinal stromal tumors (GISTs) but alone is seldom sufficient for advanced GIST. Chemotherapy and radiation therapy have no proven effect [1]. Imatinib mesylate, a tyrosine kinase inhibitor (TKI) introduced in 2000, is currently considered the first-line palliative therapy. Imatinib binds competitively to the ATP-binding pocket of KIT kinase I, which inhibits phosphorylation of tyrosine-containing substrates, downstream signaling, and cell proliferation [2]. The survival of patients with metastatic or inoperable GISTs has improved markedly with imatinib treatment [3].

Downsizing, or neoadjuvant, treatment is given to reduce tumor volume and to eradicate potential microscopic metastatic lesions prior to surgery. Such treatment in selected patients with a malignant GIST can facilitate complete resection or function-sparing surgical procedures (e.g., salvage of the anal sphincter or gastroesophageal junction in the elderly) [3]. Sunitinib, the second-line TKI, has been used for patients with mutations not responsive to 
imatinib (primary resistance), with tumor progression during imatinib treatment (secondary resistance), or with drug intolerance [4]. Sunitinib exerts antitumor activity by inhibiting the split kinase domain not only of KIT receptors but also the VEGF, PDGF, and FLT3 receptors. Furthermore, sunitinib inhibits tumor growth indirectly by inhibiting angiogenesis [5].

In vitro experiments and data from clinical trials show that the responsiveness to imatinib is dependent on the type of KIT or PDGFRA mutation [6-8]. Tumors with KIT exon 11 deletion mutation are the most sensitive to imatinib [9]. KIT mutation in GISTs can be divided into two categories: those diagnosed in primary tumors before treatment (primary mutations) and those detected during treatment with imatinib (secondary mutations) [10]; the latter can be difficult to treat [11].

Two small nonrandomized Phase II trials are currently addressing the safety and efficacy of neoadjuvant imatinib for treatment of GIST (RTOG 0132 and NCT00112632). The primary clinical endpoints are the response rate and progression-free survival. The RTOG study evaluated neoadjuvant imatinib treatment for 8 weeks before surgery and 24 months thereafter as adjuvant treatment and is now closed. The 2-year progression-free survival was $83 \%$ in a group with primary GIST and $77 \%$ in a group with recurrent or metastatic GIST [12]. The German/AustrianNCT study is still open for recruitment (40 patients planned).

The purpose of this study was twofold: (1) compare the survival of patients with high-risk resected GISTs treated with downsizing TKI versus that of historical controls from our population-based series and (2) determine if organpreserving surgery was facilitated by this treatment.

\section{Patients and methods}

Treatment group

Downsizing treatment with imatinib (400 mg/day) was given until tumor response, as judged by computed tomography (CT) would facilitate for a lesser, or more functional, operation. The downsizing study group consisted of 10 consecutive patients ( 2 women, 8 men; mean $\pm \mathrm{SD}$ age at surgery $63 \pm 8$ years, range 5575 years) with high-risk GIST (size $>5 \mathrm{~cm}$ and mitotic count $>5$; size $>10 \mathrm{~cm}$ and any mitotic count; or any size and mitotic rate $>10$ ) [13]. Seven patients had liver metastases. The mean $\pm \mathrm{SD}$ size of the primary tumors was $20.4 \pm 8.6 \mathrm{~cm}$ (range $10-35 \mathrm{~cm}$ ). One patient who was not responsive to imatinib during 3 months (progressive disease) was switched to sunitinib at a continuous dose of $37.5 \mathrm{mg} /$ day for 9 months (Table 1).
In all patients fine- or core-needle biopsies were performed for diagnosis as well as mutational analysis and determination of proliferative activity (Ki-67). The same analyses were performed on the resected tumor specimens.

After surgery the patients were continued on adjuvant TKI. The patients with metastatic disease received palliative treatment with TKI. Patients in the downsizing group were diagnosed and treated between 2002 and 2008. The study was approved by the Ethical Trial Committee at the University of Gothenburg.

\section{Control group}

The historical controls comprised 89 individuals (44 women, 45 men) who had high-risk GIST and had been subjected to R0/R2 resection [14] diagnosed during 19832000 (i.e., from a period when surgery was the only treatment and TKI had not yet been introduced); 33\% were metastatic at presentation. The controls were matched with the patients according to sex, age, tumor size and site, and maximum percent $\mathrm{Ki}-67(\max \%)$. The mean $\pm \mathrm{SD}$ age at diagnosis was $66.4 \pm 13.1$ years (range 25-92 years). The mean $\pm \mathrm{SD}$ tumor size was $14.4 \pm 8.0 \mathrm{~cm}$ (range 5$35 \mathrm{~cm}$ ), and the mean $\pm \mathrm{SD} \mathrm{Ki}-67$ max $\%$ was $15.0 \% \pm$ $14.4 \%$ (range $0.5-50.0 \%$ ) (Table 2). The historical control group and the treatment group were considered comparable as no statistically significant differences were detected in sex, age, tumor size and site, or $\mathrm{Ki}-67$ max $\%$.

\section{Mutational analysis}

Mutational analysis of $K I T$ exons 9, 11, 13, and 17 and PDGFRA exons 12 and 18 were performed with denaturating high performance liquid chromatography (dHPLC) and bidirectional direct sequencing in all cases [15].

\section{Statistics}

Time to progression-free survival was measured from the date of registration. Continuous data from the two groups were compared using the nonparametric Mann-Whitney test. Categoric data from the groups were compared using Fisher's exact test. Progression-free survival was recorded from the time of the initial diagnosis to the time of first recurrence, progression of residual tumor, or tumor-related death. Progression-free survival was calculated using the Kaplan-Meier method. Failure was defined as local or distant recurrence or progression, or death due to any cause. Differences in progression-free survival between the downsizing group and historical control groups were compared by the log-rank test. All statistical tests were two-sided. A value of $p<0.050$ was considered statistically significant. 


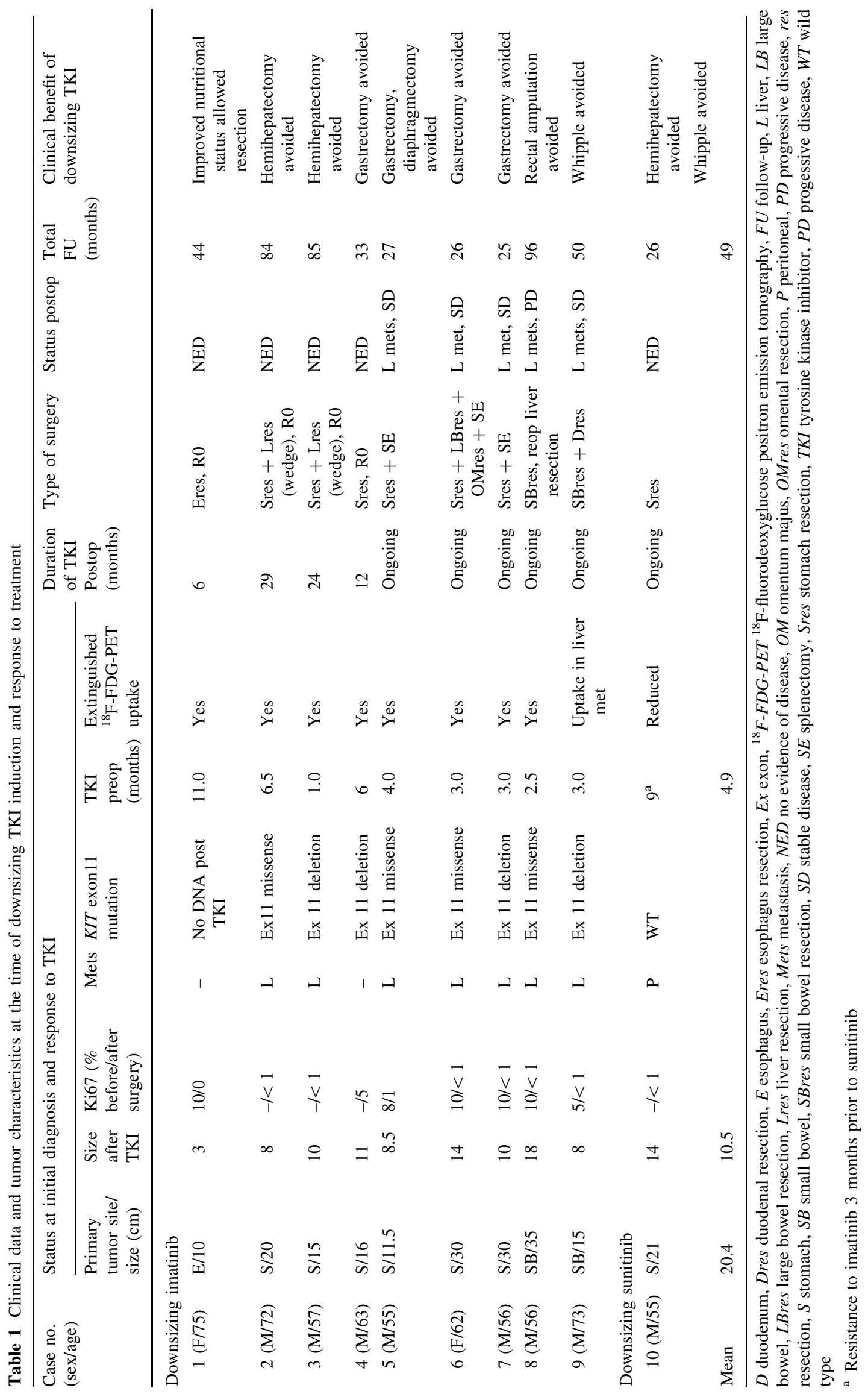




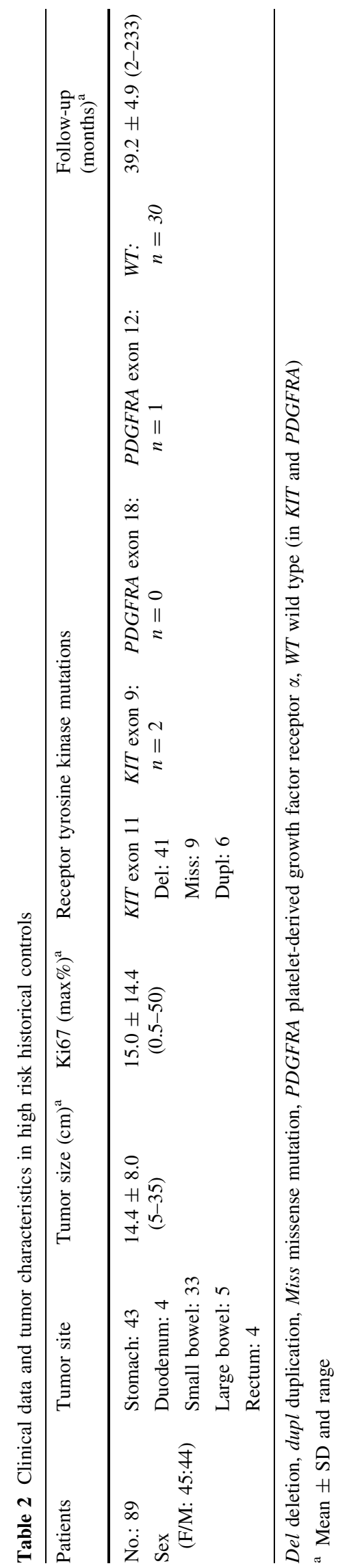

\section{Results}

Downsizing/adjuvant treatment with TKI

Ten patients were treated with downsizing TKI (Table 1, Fig. 1). The total mean follow-up after surgery of this series was $49.0 \pm 27.1$ months (range $25-90$ months). The mean duration of downsizing TKI treatment was $4.9 \pm 3.2$ months (range 1-11 months) prior to surgery followed by adjuvant imatinib at a dose of $400 \mathrm{mg} / \mathrm{day}$ for a mean period of $17.8 \pm 10.6$ months (range 6-29 months) in four patients after R0 resection; six patients with R2 resection received continuous TKI treatment after surgery. Downsizing treatment with TKI led to a mean reduction of the primary tumor of $48.5 \%$.

The patient (no. 10) with tumor progression on imatinib (3 months) was switched to sunitinib for 9 months, and the $21 \mathrm{~cm}$ tumor was reduced to $14 \mathrm{~cm}$ in diameter prior to R0 resection of the primary. The histopathologic examination after sunitinib treatment showed that the tumor was composed mainly of collagen fibrous tissue with a few tumor cells; and it was positive for KIT, DOG1, and CD34 (Fig. 2).

\section{Mutational status}

Eight of the ten patients had KIT exon 11 mutations. In one (patient 1) no DNA could be retrieved after downsizing treatment with imatinib because there was no residual viable tumor tissue, and patient 10 had a wild-type (wt) tumor (Table 1). In six patients, preoperative fine- or coreneedle biopsies showed a mean Ki-67 max\% of $8.8 \% \pm 2.0 \%$, range 5-10\%); after treatment, the surgical specimens showed a Ki-67 max\% of $\leq 1 \%(n=2), 5 \%$

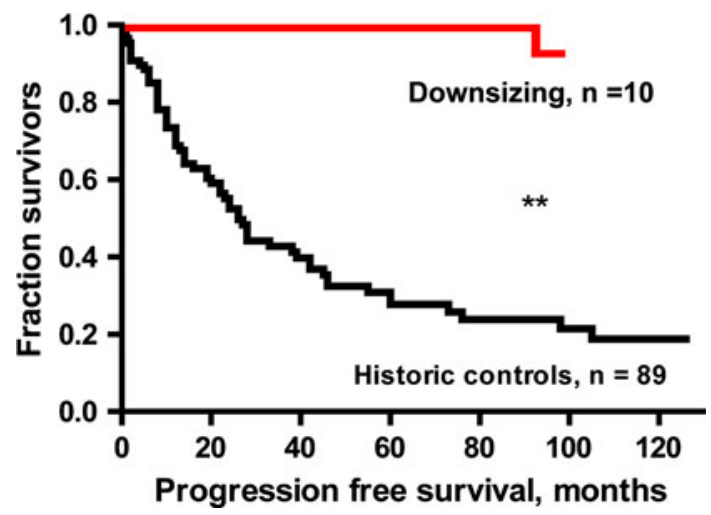

Fig. 1 Progression-free survival in high risk gastrointestinal stromal tumor (GIST) patients treated with downsizing tyrosine kinase inhibitor (TKI) until function-sparing surgery was expected (mean duration 4.9 months) prior to R0 resection of the primary followed by adjuvant TKI, compared with historical controls treated with surgery only $(* * p<0.01)$. Start date for survival was the date of diagnosis 

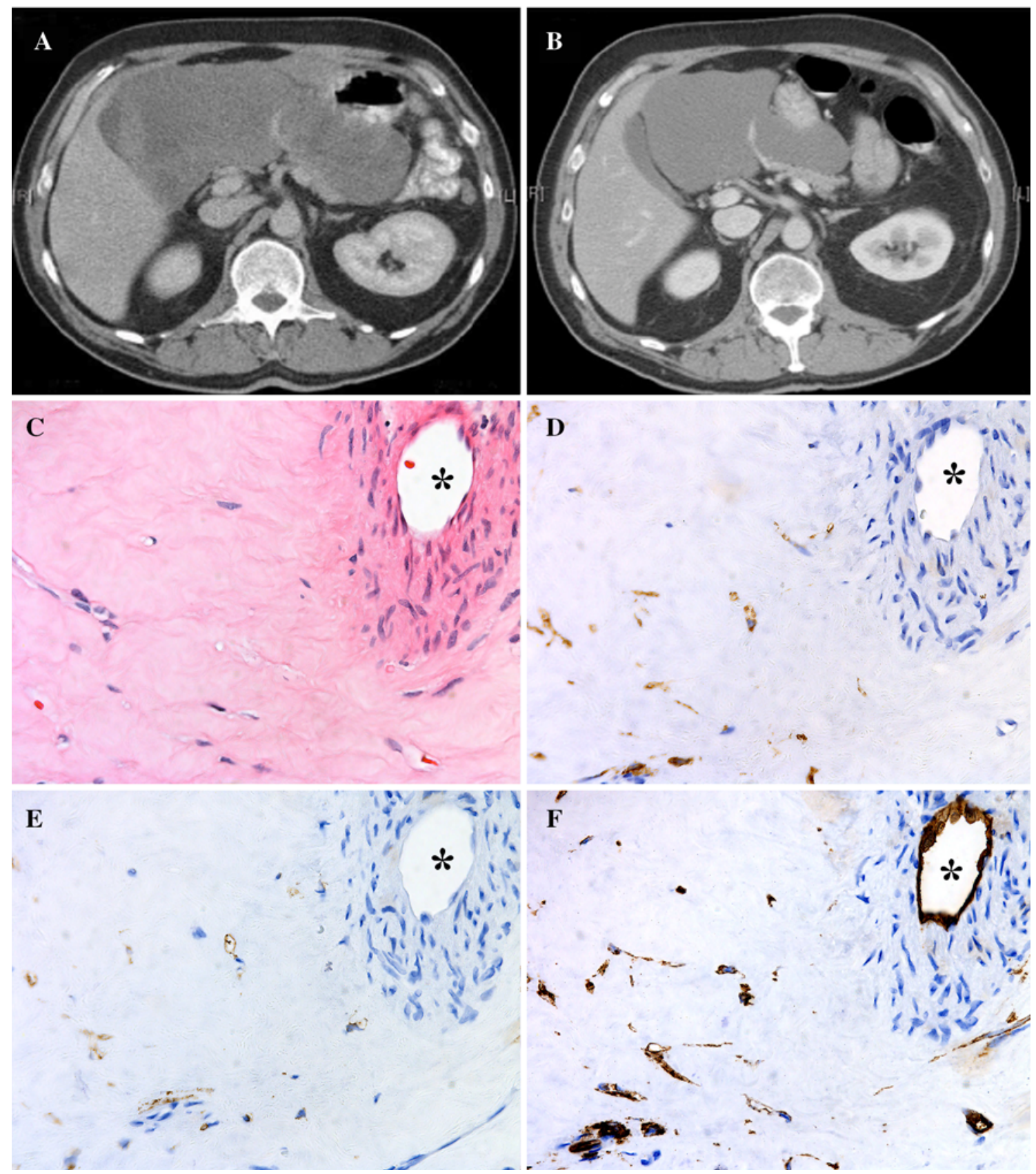

Fig. 2 Computed tomography scan before and after 9 months of downsizing sunitinib. The $21 \mathrm{~cm}$ tumor (a) was reduced to $14 \mathrm{~cm} \mathrm{(b)}$ in diameter prior to resection. c-f Histopathology of this tumor after sunitinib treatment. The tumor was composed mainly of collagen

$(n=1)$ and $<1 \%(n=3)$. In the other four cases preoperative fine-needle biopsies did not allow estimation of Ki-67 max\%.

Clinical benefit of downsizing with TKI: possibility of organ salvage

Using the downsizing strategy, eight patients treated with imatinib were able to avoid major surgical procedures: four

fibrous tissue with scattered tumor cells (c) and was positive for KIT (d), DOG1 (e), and CD34 (f). A major vessel is seen in the upper right corner $(*)$

gastrectomies (one requiring diaphragm resection), two hemihepatectomies, one Whipple procedure, and one rectal amputation. The procedures performed were limited to gastric resection, wedge resection of the liver, and salvage of the pancreas and rectum, respectively. Patient 1 had a $10 \mathrm{~kg}$ weight loss due to a relative large esophageal GIST but resumed normal nutritional status and increased her exercise capacity by $45 \%$ during 11 months of preoperative imatinib treatment. Thereafter, she underwent uneventful 
thoracoabdominal esophageal resection despite her high age.

The patient treated with sunitinib (owing to imatinib resistance) had a large gastric tumor involving the left liver lobe and pancreatic head. R0 resection of the primary lesion could be performed without hemihepatectomy or Whipple operation after a long down-staging period (Table 1).

\section{Historical controls}

The 89 historical controls, matched to high-risk GIST patients, were subjected to R0/R2 resection and had a mean follow-up of $39.2 \pm 4.9$ months (range 2-233 months). In all, 56 of these patients had tumors with KIT exon 11 mutations (41 deletions, 9 missense mutations, 6 duplications); two tumors had duplication mutations in KIT exon 9, and one tumor had a missense mutation in PDGFRA exon 12. A total of 30 patients had wild-type tumors (i.e., no mutations in KIT or PDGFRA) (Table 2).

The estimated progression-free survivals for the patients in the downsizing group and the historical control group are presented in Fig. 1. Four patients with $\mathrm{R} 0$ resection and a period of adjuvant imatinib had no recurrences versus $67 \%$ in the historical control group. Four patients with residual liver metastases have stable disease on continuous imatinib treatment after surgery, and one patient has undergone liver resection due to progressive disease after 90 months. In our population-based series [14], most recurrences of high risk GIST were seen within 2 years.

\section{Discussion}

In this study, tumor reduction of high risk GISTs by downsizing with TKI was examined. The historical group had no TKI treatment, but the study group received TKI both before and after surgery. Therefore, the improved survival in the study group must be regarded as a result of combined neoadjuvant and adjuvant TKI. The mean duration of imatinib treatment prior to surgery was 5 months, and the mean tumor reduction was about $50 \%$ during this period.

The duration of adjuvant TKI varied between 6 and 29 months (planned 24 months). The shorter treatment periods were due to adverse effects of imatinib. Patients with residual liver metastasis had continuous treatment with imatinib.

Subsequent surgery was facilitated, and R0 resection of the primary was easily performed in all cases; in two patients with liver metastases, wedge resection replaced hemihepatectomy. Of the other five patients with residual liver metastases, one was not offered liver surgery owing to age and bilobar lesions (patient 9), and two had multiple bilobar lesions (patients 5, 8); liver surgery was recommended for the other two with unilobar lesions (patients 6, 7 ), but both refused because of their good response to imatinib. One patient (no. 8) underwent liver resection after 90 months due to progression of one metastasis and currently has stable disease.

Downsizing treatment with TKI indicated that 3 to 12 months was a sufficient period to make a large primary tumor more easily resectable; only one patient (no. 3) had shorter treatment owing to the rapid effect of the downsizing treatment (1 month). Resection of involved adjacent organs or permanent intestinal stomas was avoided in this series. The treatment should be limited to specialized centers with access to mutational analyses of tumor biopsies and functional imaging with ${ }^{18} \mathrm{~F}$-fluorodeoxyglucose positron emission tomography $\left({ }^{18} \mathrm{~F}\right.$-FDG-PET), as only patients with suitable KIT mutations and objective tumor responses should be treated for longer periods with imatinib prior to surgery [4]. Patients with KIT exon 9 mutation, or wt-GIST, can be treated with sunitinib, as shown in patient 10 . The core needle biopsy indicated wt compatible with tumor progression on imatinib, which led us to change from imatinib to sunitinib.

Major surgical procedures were avoided in nine patients. Eight of these patients were treated with imatinib for 1 to 6.5 months, and another patient (no. 10) had primary treatment with imatinib for 3 months and thereafter was treated with sunitinib for 9 months. Patient 1, with a high age and poor general condition, had remarkably improved nutritional status after 11 months of downsizing with imatinib and underwent uneventful esophageal resection.

Occasional reports on the value of downsizing with TKI have appeared in the literature. Lo et al. [16] reported salvage of the rectum in a patient with high risk GIST (KIT exon 11 deletion, imatinib for 10 weeks; postoperative observation for 2 years). Ludvigsen et al. [17] reported a case similar to our patient 10 with a tumor involving the duodenum, liver, inferior caval vein, and kidney, which was downsized to the extent that a Whipple procedure with en bloc resection of the kidney could be performed (imatinib for 9 months, postoperative observation for 2 years). A case of initially, nonresectable gastric GIST was presented by Haller et al. [18]. This patient underwent R0 resection after downsizing (KIT exon 11 deletion, imatinib 10 months).

Resistance due to secondary mutations can occur during therapy with TKI, which can result in disease progression and conferred drug resistance [19]. Secondary resistance to imatinib was developed in $42 \%$ of the patients in a recent study [20]. Therefore, preoperative therapy with TKI should be given during a limited period to ensure that complete tumor resection and potential function-sparing 
surgery can be carried out. Surgery should be timed toward the period of optimal tumor response for safe surgery but before disease progression occurs [19]. Considering the case reports available, this takes place between 4 and 12 months of imatinib treatment [4]. In our series, the mean duration of downsizing treatment with imatinib was 4.9 months; only 3 of the 10 patients had treatment longer than 6 months.

Histological findings after neoadjuvant treatment are hypocellularity, myxoid degeneration with cystic change, necrosis, and scarring [21]. Residual viable KIT-positive tumors are common also in responsive patients but have low mitotic activity and show cell-cycle arrest [7]. A reduction in glycolytic metabolism can be evidenced by ${ }^{18} \mathrm{~F}$-FDGPET shortly (24-48 h) after onset of treatment [4].

Downsizing treatment with sunitinib, the second-line TKI, has not yet been evaluated in large series. Sunitinib has been used for palliation in patients with no primary response to imatinib, in those with progression on imatinib treatment due to secondary mutations, or in those who are intolerant to imatinib. Sunitinib was then most effective for GISTs that harbor KIT exon 9 mutation or wt (without KIT/ PDGFRA mutations) GISTs [22]. Patient 10 with a wt GIST showed progression on imatinib and was therefore switched to sunitinib.

Ruka et al. [23] recently reported four patients with GISTs that were initially inoperable, imatinib-resistant, or imatinib-intolerant and therefore treated with sunitinib. In two patients with $K I T$ exon 9 mutation and one with wt KIT/PDGFRA it was possible to perform R0 resection. The fourth patient (KIT exon 9 mutation) developed a dramatic partial response to sunitinib that required emergency resection of the necrotic tumor mass.

Mutational analysis is thus an important tool to select the optimal TKI for tailored downsizing in individual patients. Patients with KIT exon 13 and 17 mutations are few and seem to be resistant to available TKI, so no recommendations are at hand for these cases [11].

The optimal duration of the downsizing period should be as short as possible to avoid resistance due to selection of clones with secondary mutations (KIT exons 13 and 17) [24]. A randomized Phase II study recently reported that radiographic response and tumor cell apoptosis occur within the first week of imatinib therapy, suggesting that a short period may be enough in some patients [25]. Duration from 2 months to even more than 12 months has been published (in this study 5 months) [26]. The RTOG 0132 study used 2 to 3 months of neoadjuvant TKI [12]. A recent Phase II imatinib trial for metastatic GIST showed a median time to response of 2.7 months, but $25 \%$ of the patients did not reach partial response until 5.3 months [27], which supports our findings that some patients need more than 5 months of neoadjuvant therapy.
Acknowledgments This work was supported by grants from the Swedish Research Council, the Swedish Cancer Society, Sahlgrenska Academy (the government ALF agreement), Johan Jansson Foundation for Cancer Research, Assar Gabrielsson Research Foundation, Göteborg Medical Society, and the Royal Society for Arts and Sciences in Göteborg.

Conflict of interest None of the authors has a potential or real conflict of interest.

Open Access This article is distributed under the terms of the Creative Commons Attribution Noncommercial License which permits any noncommercial use, distribution, and reproduction in any medium, provided the original author(s) and source are credited.

\section{References}

1. DeMatteo RP, Heinrich MC, El-Rifai WM et al (2002) Clinical management of gastrointestinal stromal tumors: before and after STI-571. Hum Pathol 33:466-477

2. Tuveson DA, Willis NA, Jacks T et al (2001) STI571 inactivation of the gastrointestinal stromal tumor c-KIT oncoprotein: biological and clinical implications. Oncogene 20:5054-5058

3. Blay JY, Bonvalot S, Casali P et al (2005) Consensus meeting for the management of gastrointestinal stromal tumours: report of the GIST Consensus Conference of 20-21 March 2004, under the auspices of ESMO. Ann Oncol 16:566-578

4. Nilsson B, Andersson A, Ahlman H (2008) Adjuvant and downstaging treatment with imatinib in gastrointestinal stromal tumors. J Surg Oncol 98:145-146

5. Mendel DB, Laird AD, Xin X et al (2003) In vivo antitumor activity of SU11248, a novel tyrosine kinase inhibitor targeting vascular endothelial growth factor and platelet-derived growth factor receptors: determination of a pharmacokinetic/pharmacodynamic relationship. Clin Cancer Res 9:327-337

6. Heinrich MC, Corless CL, Demetri GD et al (2003) Kinase mutations and imatinib response in patients with metastatic gastrointestinal stromal tumor. J Clin Oncol 21:4342-4349

7. Corless CL, Schroeder A, Griffith D et al (2005) PDGFRA mutations in gastrointestinal stromal tumors: frequency, spectrum and in vitro sensitivity to imatinib. J Clin Oncol 23:5357-5364

8. Prenen H, Cools J, Mentens N et al (2006) Efficacy of the kinase inhibitor SU11248 against gastrointestinal stromal tumor mutants refractory to imatinib mesylate. Clin Cancer Res 12:2622-2627

9. Debiec-Rychter M, Sciot R, Le Cesne A et al (2006) KIT mutations and dose selection for imatinib in patients with advanced gastrointestinal stromal tumours. Eur J Cancer 42: 1093-1103

10. Lasota J, Miettinen M (2008) Clinical significance of oncogenic KIT or PDGFRA mutations in gastrointestinal stromal tumours. Histopathology 53:245-266

11. Liegl B, Hornick JL, Corless CL et al (2008) Monoclonal antibody DOG1.1 shows higher sensitivity than KIT in the diagnosis of gastrointestinal stromal tumors, including unusual subtypes. Am J Surg Pathol 33:437-446

12. Eisenberg BL, Harris J, Blanke CD et al (2009) Phase II trial of neoadjuvant/adjuvant imatinib mesylate (IM) for advanced primary and metastatic/recurrent operable gastrointestinal stromal tumor (GIST): early results of RTOG 0132/ACRIN 6665. J Surg Oncol 99:42-47

13. Fletcher CD, Berman JJ, Corless C et al (2002) Diagnosis of gastrointestinal stromal tumors: a consensus approach. Hum Pathol 33:459-465 
14. Nilsson B, Bümming P, Meis-Kindblom JM et al (2005) Gastrointestinal stromal tumors: the incidence, prevalence, clinical course, and prognostication in the preimatinib mesylate era-a population-based study in western Sweden. Cancer 103:821-829

15. Andersson J, Bumming P, Meis-Kindblom JM et al (2006) Gastrointestinal stromal tumors with KIT exon 11 deletions are associated with poor prognosis. Gastroenterology 130:1573-1581

16. Lo SS, Papacristou GI, Finkelstein SD et al (2005) Neoadjuvant imatinib in gastrointestinal stromal tumour of the rectum: report of a case. Dis Colon Rectum 48:1316-1319

17. Ludvigsen L, Toxvaerd A, Mahdi B et al (2007) Successful resection of an advanced duodenal gastrointestinal stromal tumor after downstaging with Imatinib: report of a case. Surg Today 37:1105-1109

18. Haller F, Detken S, Schulten HJ et al (2007) Surgical management after neoadjuvant imatinib therapy in gastrointestinal stromal tumours (GISTs) with respect to imatinib resistance caused by secondary KIT mutations. Ann Surg Oncol 14:526-532

19. Loughrey MB, Mitchell C, Mann GB et al (2005) Gastrointestinal stromal tumour treated with neoadjuvant imatinib. J Clin Pathol 58:779-781

20. Van Glabbeke M, Verweij J, Casali PG et al (2005) Initial and late resistance to imatinib in advanced gastrointestinal stromal tumors are predicted by different prognostic factors: a European Organisation for Research and Treatment of Cancer-Italian Sarcoma Group-Australasian Gastrointestinal Trials group study. J Clin Oncol 23:5795-5804
21. Bümming P, Andersson J, Meis-Kindblom JM et al (2003) Neoadjuvant, adjuvant and palliative treatment of gastrointestinal stromal tumours (GIST) with imatinib: a center based study of 17 patients. Br J Cancer 89:460-464

22. Chow LQ, Eckhardt SG (2007) Sunitinib: from rational design to clinical efficacy. J Clin Oncol 25:884-896

23. Ruka W, Rutkowski P, Szawlowski A et al (2008) Surgical resection of residual disease in initially inoperable imatinibresistant/intolerant gastrointestinal stromal tumor treated with sunitinib. Eur J Surg Oncol 35:87-91

24. Hohenberger P, Oladeji O, Licht A, et al. (2009) Neoadjuvant imatinib and organ preservation in locally advanced gastrointestinal stromal tumors (GIST). J Clin Oncol 27(Suppl):15 s (abstract 10550)

25. McAuliffe J, Hunt K, Lazar A et al (2009) A randomized phase II study of preoperative imatinib in GIST: evidence of rapid radiographic response and temporal induction of tumor cell apoptosis. Ann Surg Oncol 16:910-919

26. Gold J, DeMatteo R (2006) Neoadjuvant therapy for gastrointestinal stromal tumor (GIST): racing against resistance. Ann Surg Oncol 14:1247-1248

27. Blanke C, Demetri G, von Mehren M et al (2008) Long term results from a phase II trial of standard-versus higher dose imatinib mesylate for patients with unresectable or metastatic gastrointestinal stromal tumors expressing KIT. J Clin Oncol 26:620-625 